Revista de Matemática: Teoría y Aplicaciones 2003 10(1-2) : 156-167

CIMPA - UCR - CCSS ISSN: 1409-2433

\title{
EL PROBLEMA DEL CONJUNTO INDEPENDIENTE EN LA SELECCIÓN DE HORARIOS DE CURSOS
}

\author{
Rafael López Bracho* Miguel Ángel Gutiérrez-Andrade** \\ María Paula Ortuño-SÁnchez ${ }^{* * *}$ Javier Ramírez-RodríGuez ${ }^{* * * *}$
}

Recibido (versión revisada): 11 Jun 2003

\begin{abstract}
Resumen
El proceso de inscripción para alumnos de la Universidad Autónoma Metropolitana tiene como fundamento la libertad de cada alumno de seleccionar las asignaturas que cursará, así como los grupos en los que quedará inscrito. El éxito de este sistema, medido en términos del porcentaje de alumnos que obtienen inscripción en los cursos que seleccionaron, depende en gran medida tanto de las características de la oferta de grupos, relativas principalmente a la cantidad y a la variedad de horarios, como de la posibilidad por parte de los alumnos de hacer una selección adecuada de horarios para los cursos por los que optaron. Una selección adecuada de horarios de cursos es aquella en la que las asignaturas seleccionadas tienen horarios dos a dos compatibles. El problema de selección de horarios consiste en la obtención de una selección de horarios adecuada de cardinalidad máxima. En este trabajo se presentará un modelo de Teoría de Gráficas para este problema así como un algoritmo de solución para el mismo.
\end{abstract}

Palabras clave: Calendarización, Conjunto Independiente, Investigación de Operaciones, Teoría de Gráficas.

\footnotetext{
Abstract

Registration process at the Universidad Autónoma Metropolitana is such that every student is free to choose his/her own subjects and schedule. Success of this system, based in the percentage of students that obtain a place in the lectures chosen,

*Departamento de Sistemas, Universidad Autónoma Metropolitana - Unidad Azcapotzalco. Av. San Pablo 180, Colonia Reynosa Tamaulipas, 02200 México, D. F., México. E-Mail: rlb@correo.azc.uam.mx

${ }^{* *}$ Misma dirección que R. López-Bracho. E-Mail: gama@correo.azc.uam.mx

${ }^{* * *}$ Misma dirección que R. López-Bracho. E-Mail: pos@correo.azc.uam.mx

${ }^{* * * *}$ Misma dirección que R. López-Bracho. E-Mail: jararo@correo.azc.uam.mx
} 
depends principally on the characteristics of the supply of scheduled lectures, relatives to quantity and variety of timetables, as well as the oportunity of the students to do an adequate selection of lectures. An adequate selection of lectures is a subset of the lectures set with pairwise different subjects and timetables. The Choose Lectures Problem is to find the maximal adequate selection of lectures. A Graph Theory model of the problem and an algorithm to solve it will be shown.

Keywords: Graph Theory, Independent Set, Operations Research, Educational Timetabling.

Mathematics Subject Classification: 05C69, 05C85, 05C90, 68R10

\section{Introducción}

El proceso de inscripción para alumnos de la División de Ciencias Básicas e Ingeniería de la Universidad Autónoma Metropolitana Unidad Azcapotzalco tiene como fundamento la libertad de cada alumno de seleccionar las asignaturas que cursará, así como los grupos en los que quedará inscrito. El éxito de este sistema, medido en términos del porcentaje de alumnos que obtienen inscripción en los cursos que seleccionaron, depende en gran medida tanto de las características de la oferta de grupos, relativas principalmente a la cantidad y a la variedad de horarios, como de la posibilidad por parte de los alumnos de hacer una selección adecuada de horarios para los cursos por los que optaron.

\section{El proceso de inscripción}

La División de Ciencias Básicas e Ingeniería, a través de su Consejo Divisional, define para cada asignatura el número mínimo de grupos que se debe abrir en cada trimestre.

De acuerdo con estos parámetros, con base en su disponibilidad de profesores y en estadísticas internas de demanda y de reprobación, cada departamento determina el número de grupos que abrirá de cada asignatura bajo su responsabilidad, así como los cupos y horarios correspondientes, donde un horario se define por la especificación de los días y horas semanales en que se imparte el curso.

La gran diversidad de cursos a programar y la libertad que tiene cada alumno de seleccionar los grupos en los que cursará cada asignatura de su interés, imposibilitan a los coordinadores de estudio realizar una revisión exhaustiva de la pertinencia de la programación, en cuanto a las necesidades de cada estudiante en particular.

El Consejo Divisional y las coordinaciones de estudio han elaborado un diagrama del nivel de avance que recomiendan debe alcanzar cada alumno por trimestre. Este diagrama constituye un instrumento orientador para la selección trimestral de las asignaturas que debe cursar cada alumno regular y sirve también como guía para corregir las diferencias de avance de los alumnos irregulares.

Cuidar que los alumnos regulares reciban la oferta indicada en el diagrama de avance para el trimestre que les corresponde, es una labor que los coordinadores de estudio están en posibilidad de atender. 
Uno de los objetivos de este trabajo es mostrar un modelo de Teoría de Gráficas que puede ayudar a hacer el seguimiento de la oferta de cursos. Para efecto de la construcción del modelo proponemos las siguientes dos definiciones:

Definición 2.1 Dos horarios son compatibles si corresponden a diferente asignatura y su intersección es vacía (no se traslapan).

Definición 2.2 Una selección de horarios es inadecuada cuando excluye al menos una asignatura de las que originalmente se habian considerado, o cuando incluye horarios incompatibles.

La diversidad de horarios para cada curso permite pensar que los alumnos regulares no tendrán problemas para seleccionar los grupos que requieren, pero se debe considerar también que una misma asignatura puede ser cursada por alumnos de varias licenciaturas simultáneamente, por lo que se puede generar un problema de falta de cupo en algunos grupos y materias durante el proceso de inscripción, situación que puede llevar a los alumnos a una selección inadecuada de los cursos y grupos en los que finalmente quedarán inscritos.

En el proceso de inscripción, organizado por la Coordinación de Sistemas Escolares (CSE), se va atendiendo a los alumnos de acuerdo con un orden establecido en forma parcialmente aleatoria. Para obtener ese orden se siguen los pasos siguientes:

1. Se calcula para cada alumno su índice de eficiencia, el cual se obtiene por el cociente del número de créditos obtenidos por el alumno desde su ingreso, respecto al número de créditos en que ha estado inscrito en el mismo periodo.

2. Se establece un orden de prioridad basado en los índices de eficiencia, de manera que cada alumno sea inscrito antes que todos los alumnos que tengan menor índice de eficiencia que el suyo.

3. Se efectúa un sorteo para establecer el orden en que serán atendidos los alumnos que tengan la misma eficiencia.

De acuerdo con el orden establecido, los alumnos son citados por la CSE para efectuar su inscripción.

En esta parte del proceso, los alumnos presentan una solicitud que incluye las asignaturas y grupos seleccionados. Ésta es revisada por la CSE respecto a la pertinencia curricular y posteriormente se pasa al módulo de inscripción, en el cual, ante una terminal del sistema de cómputo, se da de alta una a una las materias y grupos seleccionados. Si en algún caso el grupo seleccionado no tiene cupo disponible, el alumno podrá en ese momento modificar su propuesta, pudiendo darse la situación de que el alumno ya no tenga otro grupo o asignatura por cual optar, o termine seleccionando un grupo que tenga un horario incompatible respecto a los otros horarios que ya llevaba inscritos.

Aunque al final del proceso se abre un periodo para efectuar correcciones, no es extraño que algún alumno quede insatisfecho con su inscripción, ya sea porque no pudo 
inscribirse a alguna de las asignaturas seleccionadas, o se haya inscrito en grupos con horarios incompatibles.

La construcción de un módulo de ayuda para la selección de horarios, que auxilie a los alumnos en la selección de grupos con horarios compatibles para las asignaturas de su interés, así como la implantación del módulo en el programa de inscripciones de la CSE, son acciones que pueden ayudar a mejorar la eficiencia del proceso de inscripción para los alumnos de CBI.

El modelo de Teoría de Gráficas para representar el problema de selección de horarios y el algoritmo de solución, son la base del módulo de ayuda.

\section{Modelo de Teoría de Gráficas para el problema de selec- ción de horarios de cursos.}

De acuerdo con las asignaturas que un alumno desea cursar, el problema de selección de horarios de cursos consiste en la búsqueda de un conjunto de grupos en los cuales el alumno pueda inscribirse a su entera satisfacción, entendida ésta como la posibilidad de cursar todas las asignaturas de su interés, en grupos con horarios dos a dos compatibles. Con base en esto afirmamos que:

En una instancia del problema de selección de horarios se tiene un conjunto de asignaturas $D$ y un conjunto $L$ constituido por los grupos propuestos para impartir las asignaturas en $D$. El problema consiste en determinar un conjunto de grupos $S \subseteq L$ con horarios dos a dos compatibles, de cardinalidad máxima.

Para un conjunto de asignaturas $D$ propuesto, el problema se puede modelar a través de una gráfica $G$, donde $V(G)$ y $A(G)$ denotarán el conjunto de vértices y el conjunto de aristas, respectivamente. A cada vértice se le va a asociar un grupo de alguna de las asignaturas en $D$, esto es: $V(G)=L$; mientras que dos vértices $u, v \in V(G)$ están unidos por una arista $u v \in A(G)$, si, y sólo si los horarios asociados a éstos son incompatibles.

Ejemplo 3.1 En la Figura 1 se muestran las asignaturas que en el diagrama de avance de Ingeniería Electrónica se recomienda cursar en el sexto trimestre de inscripción a dicha licenciatura. El Cuadro muestra además los horarios de los grupos que la División de Ciencias Básicas e Ingeniería abrió en el trimestre 00-primavera para las asignaturas. De acuerdo con la programación descrita, la gráfica correspondiente se muestra en Figura 2. 


\begin{tabular}{|c|c|c|c|c|c|c|}
\hline Asignatura & Grupo & Lunes & Martes & Miércoles & Jueves & Viernes \\
\hline & 1 & $7: 00-8: 30$ & & $7: 00-8: 30$ & & $7: 00-8: 30$ \\
& 2 & $8: 30-10: 00$ & & $8: 30-10: 00$ & & $8: 30-10: 00$ \\
Probabilidad y & 3 & $11: 30-13: 00$ & & $11: 30-13: 00$ & & $11: 30-13: 00$ \\
Estadística & 5 & $11: 30-13: 00$ & & $11: 30-13: 00$ & & $11: 30-13: 00$ \\
(PyE) & 6 & $16: 00-14: 30$ & & $13: 00-14: 30$ & & $13: 00-14: 30$ \\
& 7 & $20: 30-22: 00$ & & $16: 00-17: 30$ & & $16: 00-17: 30$ \\
& 8 & & $7: 00-9: 15$ & & $7: 00-9: 15$ & \\
& 9 & & $12: 15-14: 30$ & & $12: 15-14: 30$ & \\
\hline & 1 & $7: 00-8: 30$ & & $7: 00-8: 30$ & & $7: 00-8: 30$ \\
Investigación de & 2 & $8: 30-10: 00$ & & $8: 30-10: 00$ & & $8: 30-10: 00$ \\
Operaciones I & 4 & $10: 00-11: 30$ & & $10: 00-11: 30$ & & $10: 00-11: 30$ \\
(IO I) & 5 & $11: 30-13: 00$ & & $10: 00-11: 30$ & & $10: 00-11: 30$ \\
& 6 & $14: 30-16: 00$ & & $11: 30-13: 00$ & & $11: 30-13: 00$ \\
& 7 & & $18: 30-16: 00$ & & $14: 30-16: 00$ \\
\hline Electrónica II & 1 & $8: 30-10: 00$ & & $8: 30-10: 00$ & & $8: 30-10: 00$ \\
(E II) & 2 & $17: 30-19: 00$ & & $17: 30-19: 00$ & & $17: 30-19: 00$ \\
\hline Laboratorio de & 1 & & $13: 00-16: 00$ & & & \\
Electrónica II & 2 & & & & $19: 00-22: 00$ & \\
(LE II) & & & & & & \\
\hline Laboratorio de & 1 & & $7: 00-10: 00$ & & & \\
Circuitos Lógicos y & 2 & & $10: 00-13: 00$ & & $16: 00-15-20: 30$ & \\
Computadoras II & 3 & & & & & \\
(LCLyC II) & & & & & & \\
\hline Comunicaciones I & 1 & $7: 00-8: 30$ & & & & \\
(C I) & 2 & $17: 30-19: 00$ & & $17: 30-19: 00$ & & \\
\hline Laboratorio de & 1 & & & & & \\
Comunicaciones I & 2 & & $10: 00-13: 00$ & & & \\
(LC I) & 3 & & & & & \\
\hline
\end{tabular}

Figura 1: Horarios de grupos. 


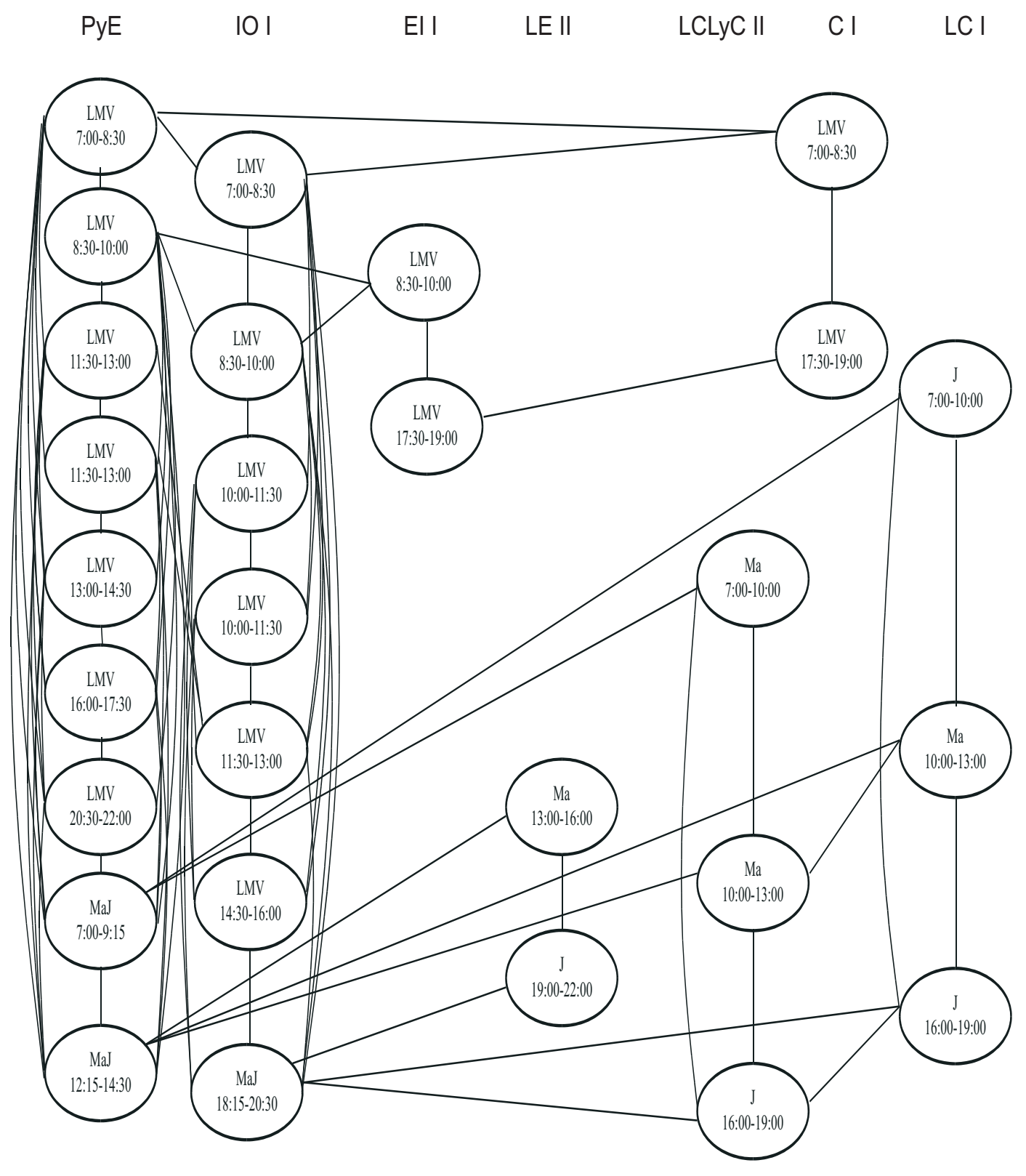

Figura 2: Gráfica de Horarios. 


\section{Características de la gráfica de horarios}

Ahora vamos a introducir algunos conceptos que nos permitirán establecer algunas propiedades de la gráfica de horarios e introducir un algoritmo adecuado para la solución del problema.

\subsection{El problema del Conjunto Independiente}

Considérese una gráfica $G$. Un conjunto $S$ de vértices de $G$ es independiente o estable si no existen dos vértices de $S$ que sean adyacentes en $G$, esto es, la subgráfica de $G$ inducida por $S$ no tiene aristas. El máximo número de vértices en un conjunto independiente de $G$ se denota por $\alpha(G)$ y se llama número de independencia o número de estabilidad de $G$.

Un conjunto independiente $\mathrm{S}$ es maximal, si para todo $v \in V(G)-S, S \cup\{v\}$ no es independiente. Claramente $|S| \leq \alpha(G)$ y la igualdad es alcanzada sólo por algunos conjuntos independientes.

El problema de determinar el número de independencia de una gráfica es un problema NP-Completo, de hecho es tan difícil determinar el número de independencia de una gráfica, como encontrar todos los conjuntos independientes maximales de la misma. Los algoritmos exactos que resuelven el primer problema resuelven en sí el segundo.

Por lo anterior, vamos a considerar que el Problema del Conjunto Independiente consiste en determinar todos los conjuntos independientes maximales de una gráfica.

\subsection{Partición de un conjunto de vértices en clanes}

Dada una gráfica $G$, se dice que un subconjunto $V_{1}$ de $V(G)$ es un clan de $G$ si la subgráfica inducida por $V_{1}$ es completa. $V_{1}, V_{2}, \ldots, V_{k}$ constituyen una partición en clanes de $V(G)$, si $V_{1}, V_{2}, \ldots, V_{k}$ forman una partición de $V(G)$ y cada $V_{i}$ es un clan de $V(G)$. $\theta(G)$ denota el número mínimo de clanes en una partición en clanes de $V(G)$.

Un resultado conocido que relaciona $\alpha(G)$ con $\theta(G)$ es el siguiente:

Teorema 4.1 [1]. Para toda gráfica $G$ se tiene que $\alpha(G) \leq \theta(G)$ y si $S$ es un conjunto independiente de vértices de $G$ y $C=\left(V_{1}, V_{2}, \ldots, V_{k}\right)$ es una partición en clanes de $V(G)$ tales que $|S|=|C|$, entonces $S$ es un conjunto independiente de cardinalidad máxima y $C$ una partición de vértices en clanes de cardinalidad mínima.

Una gráfica $G$ es una gráfica perfecta si $\alpha\left(G_{A}\right)=\theta\left(G_{A}\right)$, para toda subgráfica inducida $G_{A}$ de $G$.

Ejemplo 4.2 Un ciclo impar de longitud $2 k+1, k>1$, sin cuerdas constituye una gráfica $G$ que no es perfecta, ya que $\alpha(G)=k$ y $\theta(G)=k+1$.

Cualquier gráfica que tenga una subgráfica inducida que corresponda a un ciclo impar de longitud mayor que 3 sin cuerdas no es perfecta. 


\subsection{Conjuntos Independientes en la gráfica de horarios}

De acuerdo con los conceptos presentados en las subsecciones anteriores, existe una relación biyectiva entre los conjuntos independientes de vértices en la gráfica de horarios y los conjuntos de grupos con horarios dos a dos compatibles. Se puede determinar los conjuntos de grupos $S \subseteq L$ con horarios dos a dos compatibles de cardinalidad máxima si se resuelve el problema del conjunto independiente en la gráfica de horarios.

Si $G$ es una gráfica de horarios, una partición natural del conjunto de vértices de $G$ en clanes se obtiene de agrupar los vértices correspondientes a la misma asignatura, de donde, por Teorema 4.1, $\alpha(G) \leq|D|$, siendo $D$ el conjunto de asignaturas. Además, si los vértices de dos o más asignaturas forman un solo clan, la desigualdad anterior se cumple estrictamente.

Algoritmos eficientes para el problema del conjunto independiente han sido desarrollados para algunas gráficas específicas, como es el caso del algoritmo para gráficas CAN-Free mostrado en [9] y del algoritmo de Golumbic para gráficas perfectas, presentado en [6].

Ninguno de los algoritmos anteriores puede aplicarse a la gráfica de la Figura 2. En el caso particular del algoritmo de Golumbic, los vértices correspondientes a los grupos PyE-8, LCLyC II-1, LCLyC II-2, LC I-2 y LC I-1 forman un ciclo de longitud 5 sin cuerdas en la gráfica, por lo que ésta no es perfecta.

Técnicas heurísticas se han aplicado con éxito en la solución del problema del conjunto independiente. En [8] se muestran algunas de ellas. En el caso particular de las gráficas de horarios generadas para las asignaturas de la UAM, considerando el número de vértices que contienen, es posible aplicar con éxito algoritmos de enumeración implícita, como el algoritmo de Bron y Kerbosch [2] y el que presentan López-Bracho y Ortuño-Sánchez en [11]. El algoritmo para el problema de selección de horarios resuelve el problema del conjunto independiente en cada etapa de su aplicación.

\section{Solución del problema de selección de horarios}

El método de solución del problema de selección de horarios implica resolver repetidas veces el problema del conjunto Independiente, en una gráfica diferente en cada ocasión. Las gráficas se obtienen por medio de una sucesión de subgráficas, generadas una tras otra por la selección de un grupo que realiza el usuario (alumno) y la eliminación de todos los grupos incompatibles con éste.

Algunas propiedades y definiciones importantes para garantizar el funcionamiento del método son mostrados ahora:

Sean $S_{m}(G)$ la familia de conjuntos independientes de cardinalidad máxima de $G$ y $S_{m}(G)_{v}$ la familia de conjuntos independientes de cardinalidad máxima de $G$ que contienen al vértice $v$. Esto es $S \in S_{m}(G) \Leftrightarrow S$ es un conjunto independiente de $G$, con $|S|=\alpha(G)$; mientras que $S \in S_{m}(G)_{v} \Leftrightarrow S$ es un conjunto independiente de $G$ conteniendo al vértice $v$, con $|S|=\alpha(G)$.

Sea $\Gamma(v)$ el conjunto de vértices adyacentes a $v$ en $G$. 
Proposición 5.1 Un vértice aislado pertenece a todos los conjuntos independientes de cardinalidad máxima de una gráfica.

Prueba: Supongamos que $v$ es un vértice aislado de una gráfica $G$ y $S$ es un conjunto independiente de cardinalidad máxima de $G$ que no contiene a $v$. Obviamente $S \cup\{v\}$ es también independiente, lo que contradice el supuesto de que $S$ es de cardinalidad máxima.

Teorema 5.2 Si $S_{m}(G)_{v}$ es no vacío, entonces $S_{m}(G-\Gamma(v))_{v}$ es no vacío y $S_{m}(G-\Gamma(v))_{v}=S_{m}(G-\Gamma(v))=S_{m}(G)_{v}$.

Prueba: Claramente $S_{m}(G-\Gamma(v))_{v} \subseteq S_{m}(G-\Gamma(v))$. Considerando que cada conjunto independiente $S$ en $S_{m}(G-\Gamma(v))$ no contiene vértices de $\Gamma(v)$ y que $v$ es aislado en $G-\Gamma(v)$, por Proposición 5.1, $S$ debe contener a $v$, de donde $S_{m}(G-\Gamma(v))_{v}$ es no vacío y $S_{m}(G-\Gamma(v)) \subseteq S_{m}(G-\Gamma(v))_{v}$.

Por otra parte, considerando que cada conjunto independiente $S$ en $S_{m}(G)_{v}$ no contiene vértices de $\Gamma(v), S$ es un conjunto independiente de $G-\Gamma(v)$ tal que $|S|=\alpha(G)$. Tomando en cuenta que $\alpha(G-\Gamma(v)) \leq \alpha(G)$, se sigue que $S \in S_{m}(G-\Gamma(v))$ y $\alpha(G-\Gamma(v))=\alpha(G)$. Finalmente, supongamos que existe un conjunto independiente $S$ en $S_{m}(G-\Gamma(v))_{v}$ tal que $S \notin S_{m}(G)_{v}$, entonces $S$ no sería un conjunto independiente de cardinalidad máxima de $G$ por lo que $|S|<\alpha(G)$, contradiciendo el hecho de que $\alpha(G-\Gamma(v))=\alpha(G)$.

Teorema 5.3 Sea $V_{1}, V_{2}, \ldots, V_{k}$ una partición en clanes de $V(G)$ en la gráfica de horarios $G$. Si $\alpha(G)=k$, entonces cada clase $V_{i}$ contiene algún vértice $v$ tal que $S_{m}(G)_{v}$ es no vacío.

Prueba: Si $\alpha(G)=k$, entonces $\alpha(G)=\theta(G)$ y $V_{1}, V_{2}, \ldots, V_{k}$ es una partición en clanes de cardinalidad mínima de $V(G)$, de donde cada conjunto independiente de $G$ contiene algún vértice $v$ de cada clase $V_{i}$, para el cual $S_{m}(G)_{v}$ es no vacío.

\section{Algoritmo para el problema de selección de cursos.}

Sea $G=(V, A)$ la gráfica de horarios asociada al problema. $k$ denotará el orden de selección de cada curso y $\Gamma_{k}(v)$ el conjunto de vértices vecinos a $v$ en la gráfica $G_{k}$.

Etapa Inicial: Considérense $k=0, G_{0}=G$ y calcule $\alpha\left(G_{0}\right)$ y $\left|S_{m}\left(G_{0}\right)\right|$. Pase a la etapa intermedia.

Etapa intermedia: Considérese la gráfica $G_{k}$. Si $\left|S_{m}\left(G_{k}\right)\right| \leq t$ (ver Observación 5.4), pase a la etapa final, si no, seleccione un vértice $v$ de $G_{k}$ tal que $S_{m}\left(G_{k}\right)_{v}$ no es vacío. Tome $G_{k+1}=G_{k}-\Gamma_{k}(v), k=k+1$ y repita esta etapa.

Etapa Final (Construcción): Muestre todos los conjuntos independientes máximos de $G_{k}$. Seleccione uno de ellos como solución del problema. Fin.

Observación 5.4 La obtención de $\alpha\left(G_{0}\right)$ y los conjuntos $S_{m}\left(G_{k}\right)$ se realizan aplicando el algoritmo de solución del problema del conjunto independiente. El valor de $t$ representa el número de horarios de cursos compatibles que se desean considerar en la selección final. 
Cuadro 1: Número de soluciones de la gráfica $G_{k}$

\begin{tabular}{c|ccc}
\hline \hline K & Grupo seleccionado & $\left|S_{m}\left(G_{k}\right)_{v}\right|$ & $\left|S_{m}\left(G_{k}\right)\right|$ \\
\hline 1 & E II-1 & 724 & 1152 \\
2 & LE II-1 & 365 & 724 \\
3 & C I-2 & 217 & 365 \\
4 & LC I-1 & 93 & 217 \\
5 & IO I-5 & 12 & 93 \\
6 & LCLyC II-2 & 4 & 12 \\
7 & PyE-1 & 1 & 4 \\
\hline \hline
\end{tabular}

Ejemplo 5.5 En el Cuadro 1 se muestra una secuencia de selección de cursos para los horarios propuestos en el ejemplo 3.1. Se muestran también los valores de $\left|S_{m}\left(G_{k}\right)\right|$ y $\left|S_{m}\left(G_{k}\right)_{v}\right|$.

Observación 5.6 El sistema se implantaría para usarse cuando algunos grupos ya no cuenten con lugares disponibles, en cuyo caso el número de posibles soluciones disminuiría, aún al extremo de que un alumno no pueda encontrar lugar en todos los cursos que desea.

Observación 5.7 La solución del problema del conjunto independiente puede ser también una herramienta poderosa para determinar la factibilidad de la programación de horarios realizada para, en su caso, modificarla de ser necesario. Una situación como ésta se muestra en el ejemplo 5.8.

Ejemplo 5.8 En el mismo trimestre, la licenciatura en Ingeniería Industrial programó los horarios de los cursos correspondientes al sexto trimestre del diagrama de avance, como se muestra en la Figura 3. No es sencillo verificar en la tabla que no fue posible cursar ese trimestre, en horarios compatibles, las asignaturas recomendadas. A partir de la aplicación del algoritmo de selección de horarios se pudo detectar que al inscribirse en los únicos grupos programados de Ingeniería Eléctrica y Taller de Introducción a las Estructuras I, se estaría en imposibilidad de inscribirse a alguno de los grupos de Laboratorio de Ingeniería Eléctrica. En esa situación, los alumnos tuvieron que optar por sólo dos de las tres asignaturas mencionadas.

\section{Conclusiones}

El uso de herramientas matemáticas puede ayudar a mejorar considerablemente el funcionamiento de los sistemas. En este caso particular, en lo referente a la programación de los horarios que se ofrecen en cada trimestre, así como en la atención a los alumnos en el proceso de inscripciones, a través de la ayuda que se les puede proporcionar en la conformación de sus horarios.

Los algoritmos presentados se tienen ya programados y se han estado probando en la simulación de situaciones reales. Su funcionamiento ha sido aceptable, por lo que se está en 
posibilidad de proceder a su adaptación a los sistemas de cómputo utilizados en el proceso de inscripciones.

\section{Referencias}

[1] Bergé, C. (1970) Graphes et Hypergraphes. Dunod, París.

[2] Bron, C; Kerbosch, J. (1973) "Algorithm 457: Finding all cliques of an undirected graph", Commun. ACM 161: 575-577.

[3] Christofidès, N. (1975) Graph Theory: An Algorithmic Approach. Academic Press, New York.

[4] Füredi, Z. (1987) "The number of maximal independent sets in connected graph", Journal of Graph Theory 11(4): 463-470.

[5] Garey, M.R.; Johnson, D.S. (1979) Computers and Intractability: A Guide to the Theory of NP-Completeness. W. H. Freeman and Co. Publishers, San Francisco.

[6] Golumbic, M.C. (1980) Algorithmic Graph Theory and Perfect Graphs. Academic Press, New York.

[7] Griggs, J.R.; Grinstead, C.M., Guichard, D.R. (1988) "The number of maximal independent sets in a connected graph", Discrete Mathematics 68: 211-220.

[8] Gutiérrez-Andrade, M.A. (1991) La Técnica de Recocido Simulado. Tesis de doctorado, UNAM, México.

[9] Hammer, P.L.; Mahadev, N.V.R., de Werra, D. (1985) "Stability in CAN-free graphs", Journal of Combinatorial Theory, Series B 38: 23-30.

[10] Harary, F. (1972) Graph Theory. Addison Wesley, Reading, MS.

[11] López-Bracho, R.; Ortuño-Sánchez, M.P. (2000) "Un algoritmo paralelo para el problema del conjunto independiente", Revista de Matemática: Teoría y Aplicaciones 7(1-2): 125-134.

[12] Neumann-Lara, V. (1985) "Introducción a la Teoría de Gráficas", IV Coloquio del Departamento de Matemáticas del CINVESTAV, México.

[13] Papadimitriou, C.H.; Steiglitz, K. (1982) Combinatorial Optimization: Algorithms and Complexity. Prentice Hall Inc., Englewood Cliffs, N.J.

[14] Syslo, M.; Deo, N.; Kowalik, J. (1983) Discrete Optimization Algorithms with Pascal Programs. Prentice Hall Inc., Englewood Cliffs, N.J. 


\begin{tabular}{|c|c|c|c|c|c|c|}
\hline Asignatura & Grupo & Lunes & Martes & Miércoles & Jueves & Viernes \\
\hline \multirow{4}{*}{$\begin{array}{c}\text { Mediciones de } \\
\text { Ingeniería I (M.I. I) }\end{array}$} & 1 & $7: 00-8: 30$ & \multirow{4}{*}{$8: 30-10: 00$} & $7: 00-8: 30$ & \multirow{4}{*}{$8: 30-10: 00$} & \\
\hline & 2 & & & & & \\
\hline & 3 & $16: 00-17: 30$ & & $16: 00-17: 30$ & & \\
\hline & 4 & 19:00-20:30 & & 19:00-20:30 & & \\
\hline $\begin{array}{l}\text { Introducción a las } \\
\text { Estructuras I (I.E. I) }\end{array}$ & 1 & $7: 00-8: 30$ & & $7: 00-8: 30$ & & $7: 00-8: 30$ \\
\hline $\begin{array}{c}\text { Taller de } \\
\text { Introducción a las } \\
\text { Estructuras I } \\
\text { (T.I.E.) }\end{array}$ & 1 & & $7: 00-8: 30$ & & $7: 00-8: 30$ & \\
\hline $\begin{array}{l}\text { Ingeniería Eléctrica } \\
\text { (I.E.) }\end{array}$ & 1 & $15: 30-17: 30$ & & $15: 30-17: 30$ & & $15: 30-17: 30$ \\
\hline Laboratorio de & 1 & & & & 7:00-10:00 & \\
\hline Ingeniería Eléctrica & 2 & & & 16:00-19:00 & & \\
\hline (L.I.E.) & 3 & & & & & 16:00-19:00 \\
\hline Electrónica I (E I) & 1 & $7: 00-8: 30$ & & $7: 00-8: 30$ & & $7: 00-8: 30$ \\
\hline & 2 & $8: 30-10: 00$ & & $8: 30-10: 00$ & & $8: 30-10: 00$ \\
\hline & 3 & $11: 30-13: 00$ & & $11: 30-13: 00$ & & $11: 30-13: 00$ \\
\hline & 4 & $16: 00-17: 30$ & & $16: 00-17: 30$ & & $16: 00-17: 30$ \\
\hline & 5 & 19:00-20:30 & & 19:00-20:30 & & 19:00-20:30 \\
\hline & 6 & $20: 30-22: 00$ & & 20:30-22:00 & & $20: 30-22: 00$ \\
\hline Laboratorio de & 1 & & 7:00-10:00 & & & \\
\hline Electrónica (L.E.I) & 2 & & 10:00-13:00 & & & \\
\hline & 3 & & 16:00-19:00 & & & \\
\hline & 4 & & $19: 00-22: 00$ & & & \\
\hline & 5 & & & & 19:00-22:00 & \\
\hline
\end{tabular}

Figura 3: Horarios de grupos. 